This item was submitted to Loughborough's Research Repository by the author.

Items in Figshare are protected by copyright, with all rights reserved, unless otherwise indicated.

\title{
Development of a remote photoplethysmographic technique for human
} biometrics

PLEASE CITE THE PUBLISHED VERSION

http://dx.doi.org/10.1117/12.807360

PUBLISHER

(c) SPIE

VERSION

VoR (Version of Record)

\section{PUBLISHER STATEMENT}

This work is made available according to the conditions of the Creative Commons Attribution-NonCommercialNoDerivatives 4.0 International (CC BY-NC-ND 4.0) licence. Full details of this licence are available at: https://creativecommons.org/licenses/by-nc-nd/4.0/

\section{LICENCE}

CC BY-NC-ND 4.0

\section{REPOSITORY RECORD}

Shi, Ping, Sijung Hu, Angelos S. Echiadis, Vicente Azorin-Peris, Jia Zheng, and Yisheng Zhu. 2019. "Development of a Remote Photoplethysmographic Technique for Human Biometrics". figshare. https://hdl.handle.net/2134/22196. 


\title{
Development of a remote photoplenthysmographic technique for human biometrics
}

\author{
Ping Shi ${ }^{\mathrm{a}, \mathrm{b}}$, Sijung $\mathrm{Hu}^{* \mathrm{a}}{ }^{\text {,Angelos Echiadis }}{ }^{\mathrm{a}}$, Vicente Azorin Peris ${ }^{\mathrm{a}}$, Jia Zheng ${ }^{\mathrm{a}}$ and Yisheng Zhu ${ }^{\mathrm{b}}$ \\ ${ }^{a}$ Department of Electronic and Electrical Engineering, Loughborough University, Ashby Road, \\ Loughborough, Leicestershire LE11 3TU UK \\ ${ }^{\mathrm{b}}$ Department of Biomedical Engineering, Shanghai Jiao Tong University, 800 Dongchuan Road, \\ Shanghai 200240, China
}

\begin{abstract}
Non-contact reflection photoplethysmography (NRPPG) is being developed to trace pulse features for comparison with contact photoplethysmography (CPPG). Simultaneous recordings of CPPG and NRPPG signals from 22 healthy subjects were studied. The power spectrum of PPG signals were analysed and compared between NRPPG and CPPG. The recurrence plot (RP) was used as a graphical tool to visualize the time dependent behaviour of the dynamics of the pulse signals. The agreement between NRPPG and CPPG for physiological monitoring, i.e. HRV parameters, was determined by means of the Bland-Altman plot and Pearson's correlation coefficient. The results indicated that NRPPG could be used for the assessment of cardio-physiological signals.
\end{abstract}

Keywords: Photoplethysmography, Non-contact, Power spectrum, Recurrence plot, Bland-Altman plot

\section{INTRODUCTION}

\subsection{Advantages of Non-contact PPG}

Photoplethysmography(PPG) is a non-invasive optical technique for measuring changes in blood volume, indirectly based on variations in light intensity passing through, or reflected from, skin tissue through the use of an illumination source and a photodetector ${ }^{[1]}$. The variation in the detected output is generated by the pulsation of arterial blood within the peripheral vasculature as stimulated by the quasi-periodic cardiac cycle ${ }^{[2]}$.

The use of conventional contact PPG (CPPG) probes, whereby the light source and detector are in contact with the skin surface, means that measurements can only be taken from tissue where the probe fits. In addition, the contact probes cannot be used if mechanical isolation is required, such as the case for burned patients in an emergency room. The application of non-contact PPG could be advantageous in settings such as the cardiovascular monitoring of neonates, patients with skin trauma or for large scale population screening in which hygiene is a practical issue.

\subsection{Engineering setup}

As the detection of direct coupling is potentially unavoidable in non-contact reflection photoplethysmography (NRPPG) systems due to the separation of the illumination source and the photodetector from skin tissue, the photonics engineering setup was applied to find an appropriate relationship between illumination source and photodetector positions and orientations. The fundamental concepts underlying the design of the NRPPG system were the minimisation of direct coupling, the concentration and even distribution of source irradiance for improved signal-to-noise ratio and reduced sensitivity to motion artefact, and the subsequent detection of light constrained to an area with the aforementioned light source characteristics. Vertical cavity surface-emitting LEDs (VCSEL) have an inherently narrow beam divergence and a high coherence, both of which are advantageous in terms of concentration of irradiance. A

*S.Hu@lboro.ac.uk; phone+44 1509227059

Design and Quality for Biomedical Technologies II, edited by Ramesh Raghavachari, Rongguang Liang, Proc. of SPIE Vol. 7170, 717006

(c) 2009 SPIE - CCC code: 1605-7422/09/\$18 - doi: 10.1117/12.807360

Proc. of SPIE Vol. $7170717006-1$ 
VCSEL (type: PH85-F1P1S2, mode: $10 \mathrm{mw}, 850 \mathrm{~nm}$, Beam Divergence: $30^{\circ}$, Roithner LaserTechnik, Austria) is used as an illumination source and is positioned to illuminate the tissue area at a changeable angle. A high-speed Silicon PIN photodiode (type: S5821-03, mode: 320-1100nm, relative sensitivity: $20^{\circ}$, Hamamatsu Photonics UK Limited) with a high quantum efficiency (QE) over the spectral range is used as a photodetector in the engineering setup, positioned perpendicular to the tissue surface at a distance of $5 \mathrm{~cm}$. The photodiode is fitted with a lens which constrains its viewing angle to $10^{\circ}$. This restricts the amount of probed tissue, thus ensuring that the measuring site is within a homogeneously illuminated area, as shown in Fig. 1.

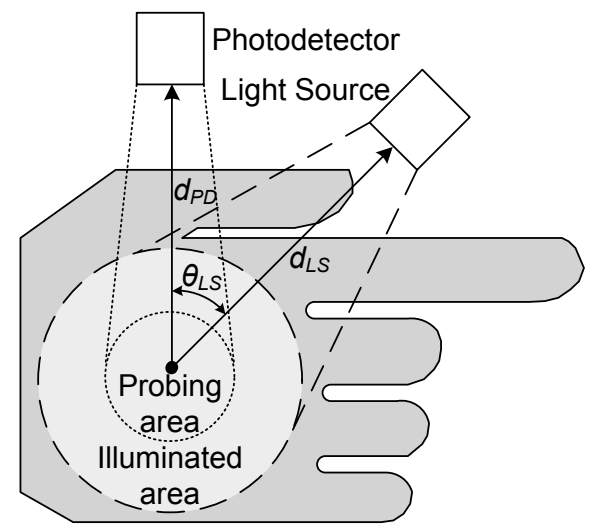

Fig. 1. Engineering setup for non-contact remote PPG illustrating the fundamental parameters

The aim of the present study is to use the developed NRPPG setup to ratify the applicability of this new technique for biomedical and clinical use.

\section{METHOD}

\subsection{Signal recording}

Twenty-two healthy subjects (M/F: 12/10, age: $29.0 \pm 9.0$ years, height: $1.70 \pm 0.10$ meter, BMI: $23.1 \pm 4.6$ ) participated in this study. Written informed consent was obtained from all volunteers prior to participation. The subjects were asked to breathe spontaneously. NRPPG signals and CPPG signals were recorded simultaneously from right index fingertip and right hand palm. Prior to data analysis, motion artifacts were minimised as the position of the hand palm was softly fixed, and ectopic beats were filtered by using Matlab (The MathWorks, Inc.).

\subsection{Spectra analysis for PPG signal}

The power spectrum of PPG signal for healthy subjects consists of three major frequency bands. They do not have fixed periods and the central frequencies may vary considerably. The limits for the spectral components, according to the heart rate of normal subjects, usually used are: VLF component $0.01-0.5 \mathrm{~Hz}$, LF component $0.5-1.5 \mathrm{~Hz}$ and $\mathrm{HF}$ component $1.5-6 \mathrm{~Hz}^{[3]}$.

Welch's method ${ }^{[4]}$ (named periodogram method) for estimating power spectra is carried out by dividing the time signal into successive blocks, and averaging squared-magnitude discrete Fourier transform of the signal blocks. The data is divided into eight segments with $50 \%$ overlap between them. The overlap between segments tends to introduce redundant information; this effect is diminished by the use of a nonrectangular window, which reduces the importance or weight given to the end samples of segments. The averaging of modified periodograms tends to decrease the variance of the estimate relative to a single periodogram estimate of the entire data record.

\subsection{Recurrence Plot}


Recurrence plots (RP) are used to reveal nonstationarity of the series. These plots were first proposed by Eckmann et al ${ }^{[5]}$ to visualize the time dependent behavior of the dynamics of systems, which can be pictured as a trajectory

$\overrightarrow{\mathrm{X}}_{\mathrm{i}} \in \mathfrak{R}^{\mathrm{n}}(\mathrm{i}=1, \cdots, \mathrm{N})$ in the $\mathrm{n}$-dimensional phase space. The main step of this visualization is the calculation of the $\mathrm{N} \times \mathrm{N}$ matrix,

$$
R_{i . j}=\Theta\left(\varepsilon_{i}-\left\|\vec{x}_{i}-\vec{x}_{j}\right\|\right) \quad i, j=1, \cdots, N
$$

Where $\varepsilon_{i}$ is a threshold distance, $\|\cdot\|$ is a norm (e.g. Euclidean norm), and $\Theta(\cdot)$ the Heaviside step function. The phase space vectors for one-dimensional time series $\mu_{i}$ from observations can be reconstructed by using the Taken's time delay method[7]

$$
\vec{x}_{i}=\left(\mu_{i}, \mu_{i+\tau}, \cdots, \mu_{i+(m-1) \tau}\right)
$$

where $m$ is the embedding dimension and $\tau$ is the time delay.

In the present study, a fixed $\varepsilon_{i}$ and the Euclidean norm are used, resulting in a symmetric RP. The binary values in $R_{i . j}$ can be simply visualized by a black and white matrix plot.

\subsection{Bland-Altman plot for agreement analysis}

Recent studies of pulse wave dynamics are reported to have strong correlations between PRV derived from PPG and HRV derived from ECG ${ }^{[6,7]}$. PRV parameters, i.e., Range of PPI, Mean of PPI, NN50, pNN50, RMSSD, SDNN, SDSD, Poincaré plot width (SD1), length (SD2) and their ratio SD1/SD2, are examined in comparison and agreement analysis. The variables from NRPPG and CPPG are described using median values with $25 \%$ and $75 \%$ interquartile range. Pearson correlation coefficients $r$ are used with respective $p$ values for analysis of the linear association between measurements based upon NRPPG and CPPG. The agreement analysis is performed according to Bland and Altman plot ${ }^{[8,9]}$. In this graphical method the differences between the two techniques are plotted against the averages of the two techniques.

\section{RESULT}

\subsection{The shape of synchronised PPG signals}

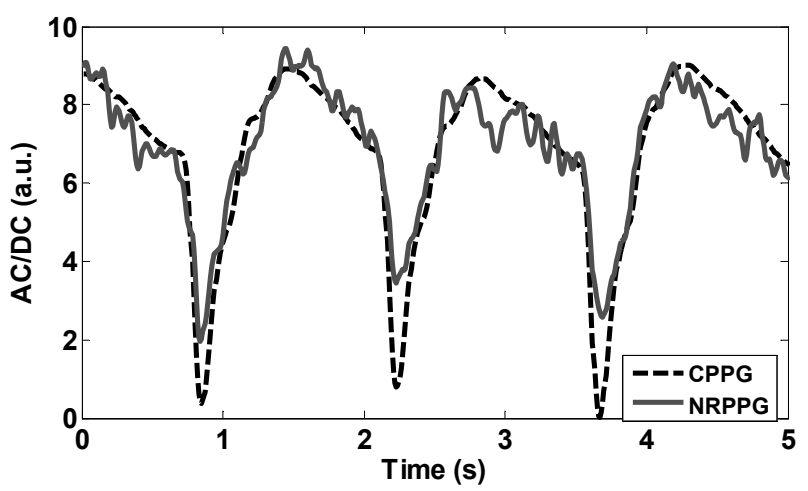

Fig. 2. Comparison of CPPG and NRPPG.

Fig.2 shows five seconds of synchronised recordings of CPPG and NRPPG intensity signals from a single subject, where an increase in light absorption during systole manifests itself as a sharp drop in signal intensity. The PPG signal 
measured from the non-contact PPG system, i.e., NRPPG, operating in reflection mode, has similar response to the PPG signal measured from the conventional contact probe.

\subsection{The spectra of PPG signals}

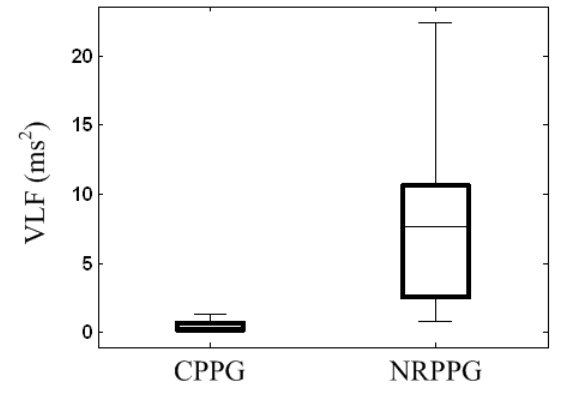

(a)

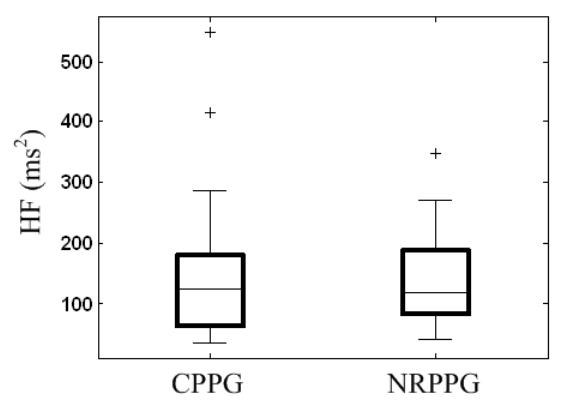

(c)

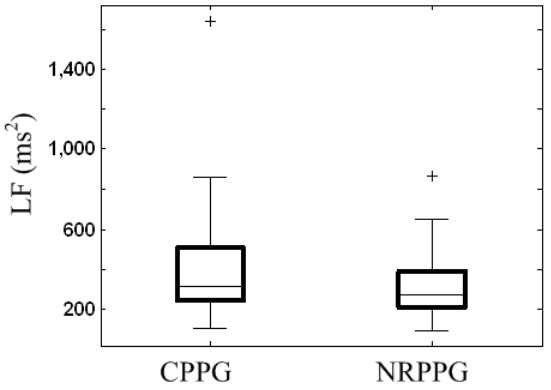

(b)

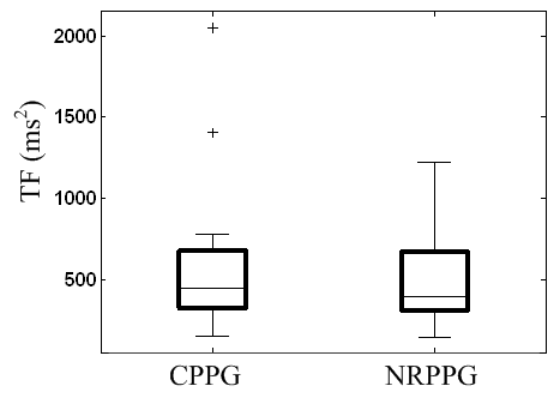

(d)

Fig. 3: The spectra of PPG signals. (a) VLF component $0.01-0.5 \mathrm{~Hz}$, (b) LF component $0.5-1.5 \mathrm{~Hz}$, (c) HF component 1.5-6 Hz and (d) total power

Fig. 3 compares the distribution of spectral regions from CPPG signals and corresponding NRPPG signals for 22 subjects. The VLF component spectra for CPPG signals have a more concentrated distribution, and are found significantly lower than those detected from NRPPG as shown in Fig. 3(a). No significant differences are found in the LF and HF components and the total power.

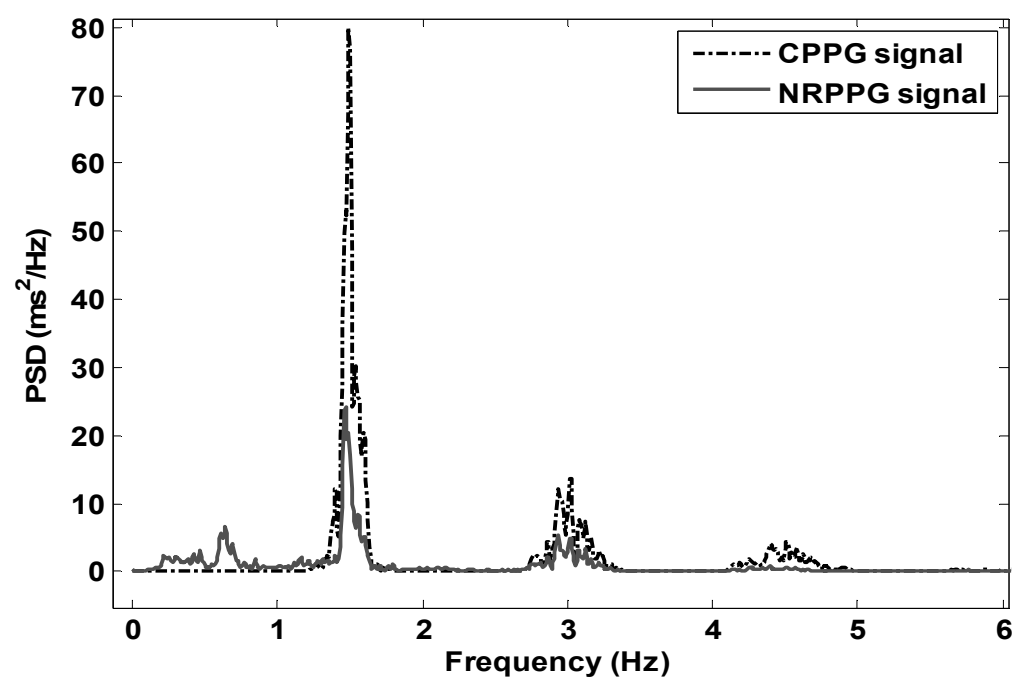

Fig. 4. Frequency spectrum of the PPG signal from a representative person. 
The frequency spectra of the PPG signals from a representative person are present in Fig. 4. CPPG signals show a higher major peak than NRPPG signals in the LF band, which is related to the beating heart. In contrast, power in the VLF band for the NRPPG signal is higher than the CPPG signal. and lower power is fond in the HF band for NRPPG.

\subsection{Recurrence plot}

The different patterns for two signals, i.e. CPPG and NRPPG, based on RP are shown, in Fig. 5. Continues diagonal line and periodic pattern are presented for CPPG signal. Horizontal and vertical lines and irregular structure are found for NRPPG signal.
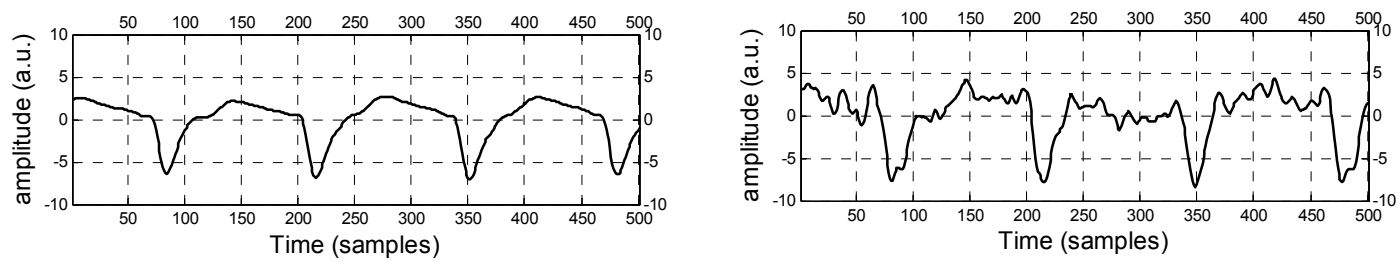

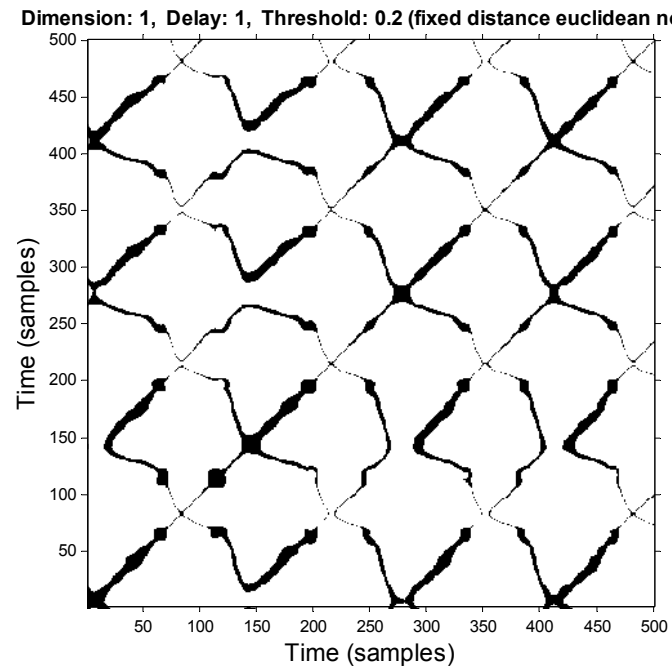

(a)

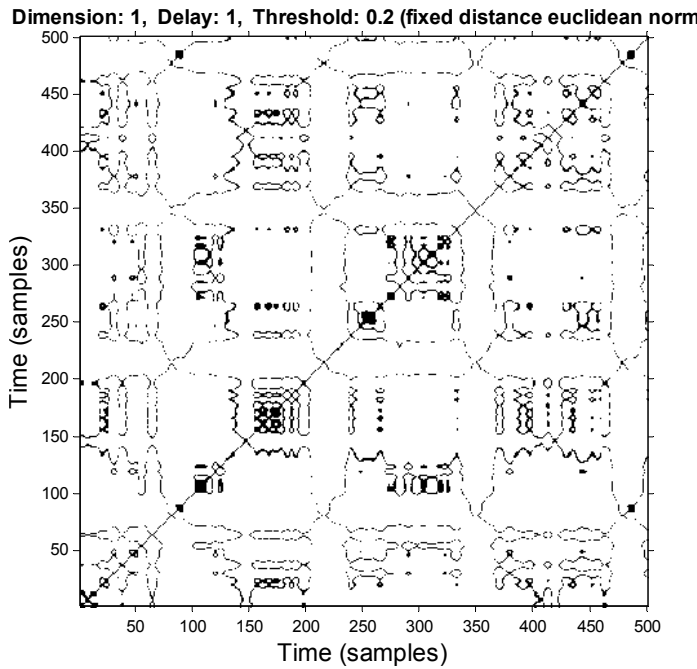

(b)

Fig. 5. Recurrence Plot is introduced as a graphical tool to tentatively study the (a) CPPG signal and (b) NRPPG signal.

\subsection{The agreement analysis of PPG signal for physiological parameters}

Table 1: The comparison and correlation relationship between CPPG and NRPPG for HR variables parameters. The values are given as median except correlation coefficients and $p$. Values in parentheses are $25 \%$ and $75 \%$ interquartile range.

\begin{tabular}{ccccc}
\hline Parameters & CPPG & NRPPG & correlation coefficients & $p$ \\
Range of $P P I(m s)$ & $250.0(179.7 \sim 365.2)$ & $269.5(169.9 \sim 371.1)$ & 0.97 & 0.0081 \\
Mean of $P P I(m s)$ & $842.3(677.6 \sim 916.7)$ & $843.1(677.1 \sim 916.4)$ & 0.99 & 0.42 \\
NN50 & $34(7.25 \sim 92)$ & $33(16.25 \sim 81.5)$ & 0.96 & 0.70 \\
pNN50 $(100 \%)$ & $0.11(0.03 \sim 0.31)$ & $0.16(0.07 \sim 0.33)$ & 0.98 & 0.016 \\
RMSSD & $35.7(24.0 \sim 49.5)$ & $42.4(28.2 \sim 56.0)$ & 0.97 & $<0.001$ \\
SDSD $(\mathrm{ms})$ & $35.8(24.1 \sim 49.6)$ & $42.5(28.2 \sim 56.1)$ & 0.97 & $<0.001$ \\
SDNN $(\mathrm{ms})$ & $39.7(25.8 \sim 60.9)$ & $40.2(30.8 \sim 62.2)$ & 0.99 & 0.002 \\
SD1 $(\mathrm{ms})$ & $21.1(15.0 \sim 35.1)$ & $26.4(19.6 \sim 39.7)$ & 0.97 & 0.0002 \\
SD2 $(\mathrm{ms})$ & $52.5(33.8 \sim 76.8)$ & $52.5(35.2 \sim 77.0)$ & 0.99 & 0.03 \\
SDI/SD2 & $0.43(0.37 \sim 0.55)$ & $0.53(0.43 \sim 0.61)$ & 0.80 & $<0.001$ \\
\hline
\end{tabular}



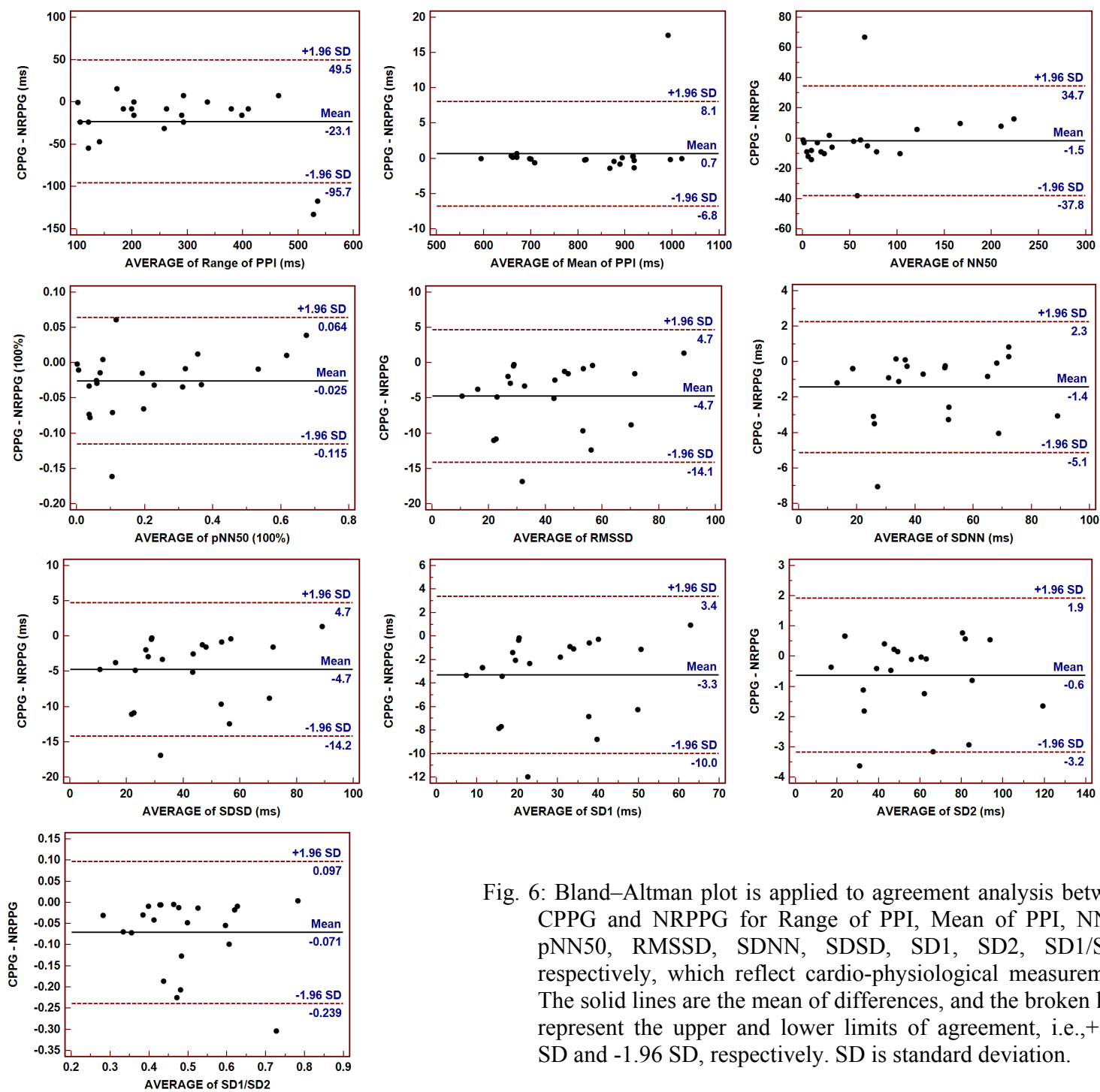

Fig. 6: Bland-Altman plot is applied to agreement analysis between CPPG and NRPPG for Range of PPI, Mean of PPI, NN50, pNN50, RMSSD, SDNN, SDSD, SD1, SD2, SD1/SD2, respectively, which reflect cardio-physiological measurement. The solid lines are the mean of differences, and the broken lines represent the upper and lower limits of agreement, i.e., +1.96 $\mathrm{SD}$ and $-1.96 \mathrm{SD}$, respectively. SD is standard deviation.

As listed in table 2, median values and interquartile ranges are similar between CPPG and NRPPG, and a strong linear association $(r>0.96)$ between the two techniques are found for all parameters except SD1/SD2. Also, as indicated in Bland-Altman plots (Fig. 6), good agreement is found for all parameters.

\section{DISCUSSION}

\subsection{PPG signal}

The PPG signals are composed of two components: a DC component, which is a relatively constant voltage, but changes in magnitude depending on the nature of the tissue through which the light passes (skin, cartilage, venous blood, etc), and an AC or pulsatile component synchronous with heart rate (HR). AC component can be divided into three parts ${ }^{[3]}$. The frequency component in the range $0.01-0.5 \mathrm{~Hz}$ reflects the activity of the sympathetic nervous system. In the frequency range $0.5-2 \mathrm{~Hz}$ the major peak depends on the individual heart rate. The information content above $2 \mathrm{~Hz}$ is due 
to the harmonics and has minimal clinical value. Attenuation of pressure and flow waves occurs as a function of distance travelled through the arterial system. In the aorta the harmonic components spread up to the 20th harmonic while at the finger capillary bed, as shown in Fig. 4, it is difficult to detect at and above the third harmonic. The higher harmonics are filtered out by the precapillary sphincter.

From the spectra analysis, the different PSD between CPPG and NRPPG signals could be attribute to the difference in probed skin, i.e. finger for CPPG and hand palm for NRPPG. The pulse shape and amplitude can vary with the relative position between the detector and the vessel under study, and that will further influence the signal PSD. Another possible explanation is that the pressure exerted on the skin by the sensor can also influence the AC signal in contact PPG ${ }^{[10]}$.

The effects of motion artefact must be considered. This includes any voluntary and involuntary movements of the interface between the probe and tissue bed. These effects can be more significant in CPPG system because the probe is more sensitive to tissue movements, especially to movements on the skin surface.

\subsection{Recurrence Plot}

The knowledge of transitions between regular or chaotic behaviours is essential to understand the underlying mechanisms behind complex systems. While several linear approaches are often insufficient to describe such processes, and most non-linear techniques, such as fractal dimensions and Lyapunov exponents, require rather long time observations. To overcome these difficulties, RP is used as graphical tool for the diagnosis of drift and hidden periodicities in the time evolution. The recurrence plot based on the vertical structures exhibits characteristic large-scale and small-scale patterns that are caused by typical dynamical behaviour ${ }^{[5]}$, e.g. continuous and periodic diagonal line in the RP diagonals, which is caused by the periodic states or related to the similar local evolution of different parts of the trajectory, can be seen in both patterns. Horizontal and vertical black lines, which indicate that the state does not change for some time, are only found in NRPPG signal as shown in Fig. 5. The pattern of RPs can be used to discriminate differences between the two signals. However, the physiological meanings of the patterns in the signals require further understanding.

\subsection{Agreement between contact PPG and non-contact PPG}

We present an analysis of the comparability of the contact and noncontact devices, with respect to the primary functions of photoplethysmography, using numerical and graphical techniques as appropriate. A good agreement is found for heart rate indices as shown in table 1 and Fig. 6. Correlation coefficients are close to 1 .Bland-Altman plots are constructed and errors between CPPG and NRPPG are distributed within reasonable ranges (Fig. 6), indicating that the NRPPG is compatible with CPPG.

\section{CONCLUSION}

Photoplethysmography is an optical bio-monitoring technique that non-invasively measures arterial pulsations in-vivo. Non-contact reflection photoplethysmography is particularly useful for situations in which contact is difficult or undesirable, for instance, in neonatal monitoring, patients with skin burn, or healing and trauma. Our present study concludes the compatibility of CPPG and NRPPG, that make NRPPG an attractive technique in the biomedical and clinical community. Clinically useful devices can however only be realized if effective solutions are found for: poor signal-to-noise ratio, motion artifacts, lack of dynamic range and tissue glare.

\section{ACKNOWLEDGEMENT}

The authors would like to express their thanks to Loughborough University and Shanghai Jiao Tong University for the financial support to carry out this study.

\section{REFERENCES}

[1] Hertzman, A.B. and Spealman, C.R., "Observations on the finger volume pulse recorded photoelectrically," Am J Physiol. Meas., 119, 334-335 (1937). 
[2] Nitzan, M., Babchenko, A., Khanokh, B. and Landau, D., "The variability of the photoplethysmographic signal--a potential method for the evaluation of the autonomic nervous system," Physiol. Meas., 19, 93-102 (1998).

[3] Kamal, A.A., Harness, J.B., Irving, G. and Mearns, A.J. "Skin photoplethysmography—a review," Comp. Method. Prog. Biomed., 28(4), 257-269.(1989)

[4] Welch, P.D., "The use of fast Fourier transforms for the estimation of power spectra: A method based on time averaging over short modified periodograms," IEEE Trans. on Audio and Electroacoustics," 15, 70-73 (1967).

[5] Eckmann, J.P., Kamphorst, S.O. and Ruelle, D., "Recurrence plots of dynamical systems," Europhys Lett, 4, 973977 (1987)

[6] Kageyama, T., Kabuto, M., Kaneko, T. and Nishikido, N., "Accuracy of pulse rate variability parameters obtained from finger plethysmogram: a comparison with heart rate variability parameters obtained from ECG," J. Occup. Health, 39, 154-155 (1997)

[7] Shi, P., Hu, S. and Zhu, Y., "A preliminary attempt to understand compatibility of photoplethysmographic pulse rate variability with electrocardiogramic heart rate variability”, J. Med. Biol. Eng., 28(4), 173-180 (2008)

[8] Bland, J.M. and Altman, D.G., "Statistical method for assessing agreement between two methods of clinical measurement" The Lancet, 1, 307-310 (1986)

[9] Bland, J.M. and Altman, D.G., 1999 Measuring agreement in method comparison studies Stat. Methods Med. Res., 8, 135-160, (1999)

[10] Teng, X.F. and Zhang, Y.T., "The effect of contacting force on photoplethysmographic signals", Physiol. Meas., 25(5), 1323-1335 (2004) 